Pacific Journal of Mathematic 


\title{
CONSTRUCTIVE PROOF OF THE MIN-MAX THEOREM
}

\author{
GeORGE B. DANTZIG
}

1. Introduction. The foundations of a mathematical theory of "games of strategy" were laid by John von Neumann between 1928 and 1941." The publication in 1944 of the book "Theory of Games and Economic Behavior" by von Neumann and Morgenstern climaxed this pioneering effort. The first part of this volume is concerned with games with a finite number of pure strategies with particular emphasis on the "zero-sum two-person" type of game. There it is shown that in most instances a player is at a disadvantage if he always plays the same pure strategy and that it is better to "mix" his pure strategies by some chance device. The starting point of all discussions of this type of game is the celebrated "Main Theorem" or Min-Max Theorem which is concerned with existence and properties of optimal mixed strategies for both players.

The first proofs of this theorem, given by von Neumann, made rather involved use of topology, functional calculus, and fixed point theorems of L. E. J. Brouwer. The first proof of an elementary character was given by J. Ville, 1938. The von Neumann-Morgenstern book, with the purpose of having a proof which is accessible to a less highly trained group, carries the theme of elementarization further [6]. At this late date there still continues to be a need for a truly elementary proof ; for example, the recent book of McKinsey on game theory [5] omitted a self-contained proof because none was available.

Kuhn [4] gives a bibliography of some of the better known proofs of the Min-Max Theorem, together with a discussion of their general characteristics which he broadly classifies into (1) those based on separation properties of convex sets and (2) those using some notion of a fixed point of a transformation. Kuhn [4] and McKinsey [5] provide proofs along the lines of von Neumann [6] based on a separation theorem. Dresher [3] gives a self-contained proof along the lines of Ville. As was pointed out in [7], the Min-Max Theorem is completely algebraic and should be given an algebraic proof. The purely algebraic proofs, when made selfcontained and elementary, appear to be quite long, [3], [4], [7], and, with the exception of Weyl's proof [7], make use of nonalgebraic concepts as the minimum of a continuous function on a closed bounded set is assumed on the set. All these proofs are either pure existence proofs

Received January 15, 1954 and in revised form September 29, 1954.

The author wishes to express his indebtedness to Melvin Dresher for his encouragement and suggestions.

${ }^{1}$ For the contributions of Borel to this field see Econometrica, Vol. 21, No. 1, January, 1953, pp. 95-127. 
or, from the viewpoint of practical computations, nonconstructive.

The present proof has the following features: It is purely algebraic (in the spirit of Weyl) and elementary in the sense that it used nothing more advanced than the notion of an inverse of a matrix. It is short, self-contained, and noninductive. The very nature of the solution, if desired, could be used to advantage to establish well-known theorems regarding the structure of the class of optimal strategies. It is a special adaptation for games of the simplex method used for solving linear programming problems [1]..$^{2}$ As such, it provides perhaps the most efficient means currently available for explicitly constructing optimal mixed strategies for both players.

2. The Min-Max Theorem. It has been found convenient in a part of the proof to compare certain vectors "lexicographically." The term is borrowed from an alphabetical ordering of words (as in a dictionary). Thus a vector $A$ is greater than $B$ (written $A>B$ ) if $(A-B)>0$; where by $(A-B)>0$ is meant that $(A-B)$ has nonzero components, the first of which is positive.

Let $\left[a_{i j}\right]$ be the payoff matrix of a finite zero-sum two-person game where $a_{i j}$ is the payoff to player I (the maximizing player) when player I plays pure strategy $i$ and player II (the minimizing player) plays pure strategy $j$. Player I (in order to guard against his strategy being " found out") chooses a mixed strategy $\left(x_{1}, x_{2}, \cdots, x_{m}\right)$ where $x_{i}$ is the probability of playing strategy $i$; accordingly, player I's expected payoff becomes $\left(\sum_{i} a_{i j} x_{i}\right)$ if the minimizing player plays pure strategy $j$. If player I's mixed strategy is found out he can expect that player II will choose $j$ such that $\sum_{i} a_{i j} x_{i}$ is minimum. Thus, player I wishes to choose his $x_{i}$ such that the smallest such sum (which we will denote by $x_{0}$ ) is a maximum. For similar reasons player II chooses a mixed strategy $y_{1}$, $y_{2}, \cdots, y_{n}$ such that the largest sum $\sum_{j} a_{i j} y_{j}$ (denoted by $y_{0}$ ) is minimum. The Min-Max Theorem states that there exists a choice for player I of $x_{i}=\hat{x}_{i}$ and a choice for player II of $y_{j}=\hat{y}_{j}$ such that the corresponding $x_{0}=\hat{x}_{0}$ is the maximum value for $x_{0}$ and the corresponding $y_{0}=\hat{y}_{0}$ is the minimum value for $y_{0}$ and, moreover, $\hat{x}_{0}=\hat{y}_{0}$. The common value of $\hat{x}_{0}$ and $\hat{y}_{0}$ known as the "value" of the game.

To establish this result we shall consider, as is often done, a related linear inequality problem. Let $x_{i}$ and $y_{j}$ satisfy the system of relations

2 That the simplex method itself could be used to prove the Min-Max Theorem was first pointed out by Dorfman (and H. Rubin) [2]. This paper, by incorporating methods for avoiding "degeneracy" and "cycling" in the simplex algorithm [1], puts the proof on a completely rigorous foundation. 

(1) $x_{i} \geqq 0$,
$(i=1, \cdots, m)$
(4) $y_{\jmath} \geqq 0$,
$(j=1, \cdots, n)$
(2) $\sum_{i=1}^{m} x_{i}=1$
(5) $\sum_{j=1}^{n} y_{j}=1$
(3) $\quad x_{0} \leqq \sum_{i=1}^{m} x_{i} \alpha_{i j}, \quad(j=1, \cdots, n)$;
(6) $\quad \sum_{j} a_{i}, y_{j} \leqq y_{0}, \quad(i=1, \cdots, m)$.

If we multiply (3) through by any $y_{j}$ satisfying (4), (5), and (6) and sum with respect to $j$; similarly multiply through (6) by any $x_{i}$ satisfying (1), (2), (3) and sum with respect to $i$, one obtains immediately

$$
x_{\mathrm{v}}=x_{\mathrm{v}} \sum_{j} y_{j} \leqq \sum_{i} \sum_{j} x_{i} a_{i j} y_{j} \leqq y_{0} \sum_{i} x_{i}=y_{0}
$$

so that the lower bounds $x_{0}$ never exceed the upper bounds $y_{0}$.

We shall, however, construct a solution $x_{i}=\hat{x}_{i}$ and $y_{j}=\hat{y}_{j}$ with the property that

$$
\hat{x}_{0}=\hat{y}_{0} \text {. }
$$

In particular (7) holds for $\hat{y}_{\text {, }}$ and any $x_{0}$ and also for $\hat{x}_{0}$ and any $y_{0}$. It follows, therefore, that $x_{0} \leqq \hat{y}_{0}=\hat{x}_{0} \leqq y_{0}$ and

$$
\hat{x}_{0}=\max x_{0} \text { and } \hat{y}_{0}=\min y_{0}
$$

and the Min-Max Theorem would be demonstrated.

3. Proof of the Min-Max Theorem. We shall begin the proof by augmenting the matrix of the game $a_{i j}$ and consider the matrix

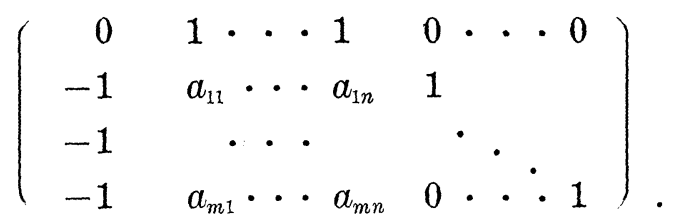

The columns of this matrix will be denoted $P_{0} ; P_{1}, \cdots, P_{n} ; P_{n+1}=U_{1}, \cdots$, $P_{n+m}=U_{m}$ where $U_{i}$ are unit vectors with 1 as the $(i+1)$ st component. It will be convenient to arrange the rows of the matrix such that

$$
a_{m 1}=\max _{i} a_{i 1} \text {. }
$$

Let $B$ (which we will call a basis) be a subset of $m+1$ columns of (10) (including $P_{0}$ as first column) which, considered as an $m+1$ square matrix, is nonsingular and let the rows of $B^{-1}$ be denoted by $\beta_{i}(i=0,1$, $\cdots, m)$. We shall further require that $B$, to be a basis, have the property that each row (except $i=0$ ) of $B^{-1}$ have its first nonzero component positive. Thus we are assuming in the lexicographic sense that

$$
\beta_{i}>0 \quad(i=1,2, \cdots, m) .
$$


For example, we may choose $B=B_{0}$ as consisting of the first two columns of (10) and the unit vectors $U_{1}, \cdots, U_{m-1}$. This near identity matrix

$$
B_{0}=\left[P_{0}, P_{1}, U_{1}, \cdots, U_{m-1}\right]=\left[P_{0}, P_{1}, P_{n+1}, \cdots, P_{n+m-1}\right]
$$

is obviously non-singular and possesses a simple inverse

$$
B_{0}^{-1}=\left(\begin{array}{cccccr}
a_{m 1} & 0 & 0 & & 0 & -1 \\
1 & 0 & 0 & . & 0 & 0 \\
b_{1} & 1 & 0 & . & \cdot & -1 \\
b_{2} & 0 & 1 & \cdot & . & \cdot \\
\cdot & \cdot & \cdot & . & . & . \\
b_{m-1} & 0 & 0 & & 1 & -1
\end{array}\right)
$$

where $b_{i}=a_{m 1}-a_{i 1}$. Because of (11) it follows that $b_{i} \geqq 0$ and our special lexicographic assumption (12) holds.

Let the columns of a general basis be denoted by

$$
B=\left[P_{0}, P_{j_{1}}, \cdots, P_{j_{m}}\right]
$$

and note that the conditions $\beta_{k} P_{j_{i}}=0$ for $i \neq k$ and $\beta_{i} P_{j_{i}}=1$ for $i, k=0$, $1, \cdots, m\left(j_{0}=0\right)$ must hold between $B$ and its inverse. The 0 -row of $B^{-1}$ is used to compute the scalar quantities $\beta_{0} P_{j}$ for $j=1,2, \cdots, n, \cdots, n+m$. We shall now prove the following.

THEOREM. If for all $j=1,2, \cdots, n+\hbar$ we have

$$
\beta_{0} P_{j} \leqq 0,
$$

then the components of the 0-row and 0-column of $B^{-1}$ yield the required optimal strategies.

Proof. Denote the components of the 0 -row of $B^{-1}$ by

$$
\left[\hat{x}_{0},-\hat{x}_{1}, \cdots,-\hat{x}_{m}\right] ;
$$

the components of the 0 -column of $B^{-1}$ by

$$
\left\{\hat{y}_{0}, \hat{y}_{j_{1}}, \cdots, \hat{y}_{j_{m}}\right\} \text {. }
$$

We shall now show that an optimum mixed strategy for player I is obtained by setting $x_{i}=\hat{x}_{i}$ for $i=1,2, \cdots, m$; and one for player II, by setting $y_{j_{i}}=\hat{y}_{j_{i}}$ for $j_{i} \leqq n$ and $y_{j}=\hat{y}_{j}=0$ for all other $j \leqq n$. Moreover, the value of the game is $\hat{x}_{0}=\hat{y}_{0}$. Indeed, for player $I$, it is easy to verify the condition $\beta_{0} P_{0}=1$ is the same as $(2)$; moreover, $\beta_{0} P_{j} \leqq 0$ for $1 \leqq j \leqq n$ are the same as (3), while for $n+1 \leqq j \leqq n+m$ they are the same as (1). For player II, the lexicographic property of the rows of $B^{-1}$, namely 
$\beta_{i}>0$ for $i=1, \cdots, m$ implies that the first component of $\beta_{i}$ (which by definition is $\hat{y}_{j_{i}}$ ) is nonnegative ; thus, (4) is satisfied. Multiplying $B$ on the right by 0 -column of $B^{-1}$ yields $(m+1)$ linear expressions in $\left(\hat{y}_{0}, \hat{y}_{j_{1}}\right.$, $\cdots, \hat{y}_{j_{m}}$ ) which may be equated to unit vector $U_{0}$.

The first of these $(m+1)$ linear equations yields (5) since the 1st components of $P_{j_{i}}$ are unity for $1 \leqq j_{i} \leqq n$ and zero otherwise. The remaining $m$ equations yield the inequalities (6) if the terms involving $j_{i}>n$ are dropped (the latter are nonnegative because $\hat{y}_{j_{i}} \geqq 0$ and their coefficients are the components of the unit vectors $\left.P_{n+i}\right)$. Finally, the proof is completed by noting that (8) or $\hat{x}_{J}=\hat{y}_{0}$ holds since both are defined in (16) and (17) as the $(0,0)$ element of $B^{-1}$.

Constructing an Optimal Basis. It is clear now that the central problem is one of constructing a basis $B$ with the property that $\beta_{0} P_{j} \leqq 0$ for $j=1,2, \cdots, n+m$ since this in turn yields an optimal mixed strategy for each player. We shall show that if some basis $B$, such as $B_{0}$, does not have the requisite property (15), then it is easy to construct from $B$ a new basis $B^{*}$ which differs from $B$ by only one column where 0 -row of $\left[B^{*}\right]^{-1}$ (which we denote by $\beta_{0}^{*}$ ) has the property that

$$
\beta_{0}>\beta_{0}^{*}
$$

that is, the first nonzero component of $\left(\beta_{0}-\beta_{0}^{*}\right)$ is positive. If the new basis $B^{*}$ does not satisfy (15) then the algorithm just outlined for $B$ is iterated, with $B$ replaced by $B^{*}$, etc. This process generates a sequence of bases which terminates when a basis is obtained that has the required property. This must occur in a finite number of steps since the condition (18) is a strict inequality which insures that no basis can be repeated and the number of different bases cannot exceed the number of ways of choosing $m$ columns out of $n+m$ from (10). ${ }^{3}$ The 0 -column of successive bases of the iterative process may be interpreted as a succession of improved mixed strategies for player II for which his expected loss, $y_{0}$, if his opponent is playing optimally, is decreasing to a minimum. Indeed, the components of the first column of any basis (as in (17) and sequel) satisfy (4) and (5) independently of condition (15), while $y_{0}$, the first component of $\beta_{0}$, is nonincreasing from basis to basis by virtue of (18).

To construct $B^{*}$ from $B$ let $P_{s}$ denote the column of (10) which replaces the $r$ th column of $B$ where $P_{s}$ and $P_{j_{r}}$ are determined by the following rules: Choose $P_{s}$ such that

3 In practical computations with the simplex method, of which this is a variation, the number of iterations is usually very small. In a game case where, say, $m / 2$ of pure strategies are used with positive probability in an optimal mixed strategy, something in the order of $m / 2$ iterations might be expected before an optimal basis is obtained. 


$$
\beta_{0} P_{s}=\max _{j} \beta_{0} P_{j}>0, \quad(j=1, \cdots, n+m) .
$$

In case the choice of $s$ is not unique, then choose $s$ with the smallest index satisfying (23). Next, compute the column vector $V$ satisfying $B V=P_{s}$. It is clear that components of $V=\left\{v_{0}, v_{1}, \cdots, v_{m}\right\}$ are given by

$$
v_{i}=\beta_{i} P_{s}
$$

$$
(i=0, \cdots, m)
$$

where, in particular $v_{0}=\beta_{0} P_{s}>0$ from (19). We now choose to drop from $B$ that column $P_{i_{r}}$ such that the lexicographic minimum of the vectors $\left(1 / v_{i}\right) \beta_{i}$ for $v_{i}>0$ is attained for $i=r$. Thus,

$$
\frac{1}{v_{r}} \beta_{r}=\min \frac{1}{v_{i}} \beta_{i}
$$$$
\left(v_{r}>0, v_{i}>0\right)
$$

where $i, r \neq 0$, and where it is assumed for the moment that there is at least one $v_{i}>0$. The minimizing vector is easily obtained in practice by finding the vector whose first component is the least; if there is a tie, then one passes to the second components of the tying vectors and selects the least, etc. A relation which will be used later that follows from (21) is

$$
\beta_{i}-\frac{v_{i}}{v_{r}} \beta_{r}>0
$$

It is clear from the structure of the augmented matrix (10) that the first column $P_{0}$ can not be formed as a positive linear combination of the other columns $P_{j}$. However, if we assume, contrary to the assumption of $(21)$, that all $v_{i} \leqq 0,(i \neq 0)$ and write

$$
P_{s}=B V=v_{0} P_{0}+\sum v_{i} P_{j_{i}},
$$

then, by transposing to the left all terms other than $v_{0} P_{0}$, we obtain a positive linear combination of columns $P_{s}$ and $P_{j_{i}}$ that yields $v_{0} P_{0}$, where $v_{0}>0$; a contradiction.

There remains only to show that $B^{*}$ has the requisite properties (12) and (18). The proof, as well as the efficiency of the computational algorithm, is obtained by constructing $\left[B^{*}\right]^{-1}$ from $B^{-1}$ using the relations

$$
\begin{aligned}
& \beta_{i}^{*}=\beta_{i}-\frac{v_{i}}{v_{r}} \beta_{r}, \\
& \beta_{r}^{*}=+\frac{1}{v_{r}} \beta_{r}
\end{aligned}
$$

where $\beta_{i}^{*}$ is the $i$ th row of $\left[B^{*}\right]^{-1}$. To verify that $(23)$ is indeed the inverse of $B^{*}$, one notes from (23) that for $i \neq r$ the values $\beta_{k}^{*} P_{j_{i}}$ are 
the same as $\beta_{k} P_{j_{i}}=0$ (or 1 if $i=k$ ); moreover, it follows readily from the definitions of $v_{i}$ given in (20) that $\beta_{r}^{*} P_{s}=1$ and $\beta_{i}^{*} P_{s}=0$ for $i \neq r$.

The required properties of $\beta_{i}^{*}$ are immediately evident: Thus, the first nonzero component of $\beta_{r}^{*}$ is positive because $\beta_{r}$ has this property and $v_{r}>0$. Next, for all other $i=1,2, \cdots, m$ the property must hold if $v_{i} \leqq 0$ since $\beta_{i}^{*}$ is the sum of two vectors with this property. If $v_{i}>0$ then $\beta_{i}^{*}>0$ by (22) and (23). Finally we note that the relation $\beta_{0}>\beta_{0}^{*}$ (and not $\beta_{0} \geq \beta_{0}^{*}$ ) holds because $\beta_{r}$, a row of a nonsingular matrix, possesses at least one nonzero component and $\beta_{0}^{*}$ is formed by subtracting from $\beta_{0}$ a vector $\left(v_{0} / v_{r}\right) \beta_{r}$ where $v_{0}>0, v_{r}>0$; hence, (18) holds and the proof is complete.

4. Example. Solve the $3 \times 6$ game matrix $M$

$$
M=\left(\begin{array}{llllll}
4 & 3 & 3 & 2 & 2 & 6 \\
6^{*} & 0 & 4 & 2 & 6 & 2 \\
0 & 7 & 3 & 6 & 2 & 2
\end{array}\right)
$$

from [8, Chap. 3, Ex. 10]. Element $a_{21}$ of $M$ has been starred. It will be noted that this is the maximal element in the first column. For convenience, see (11), the second and third rows have been interchanged so that this element appears in the bottom position of this column in forming the augmented matrix, $\left[P_{0}, \cdots, P_{9}\right]$, given below:

$$
\left(\begin{array}{cccccccccc}
P_{0} & P_{1} & P_{2} & P_{3} & P_{4} & P_{5} & P_{6} & P_{7} & P_{8} & P_{9} \\
0 & 1 & 1 & 1 & 1 & 1 & 1 & & & \\
-1 & 4 & 3 & 3 & 2 & 2 & 6 & 1 & & \\
-1 & 0 & 7 & 3 & 6 & 2 & 2 & & 1 & \\
-1 & 6 & 0 & 4 & 2 & 6 & 2 & & & 1
\end{array}\right) .
$$

Initial Iteration. The initial basis, $B=B_{0}$, consists of $P_{0}, P_{1}, P_{7}=U_{1}$, $P_{8}=U_{2}$. The inverse of $B_{0}$ (given below) is determined by formula (13). The entries $v_{i}$ shown, for the moment, cannot be filled in until $P_{s}$ is first determined.

$$
B_{0}^{-1}=\left(\begin{array}{c}
\beta_{0} \\
\beta_{1} \\
\beta_{2} \\
\beta_{3}
\end{array}\right)=\left(\begin{array}{rrrr}
6 & 0 & 0 & -1 \\
1 & 0 & 0 & 0 \\
2 & 1 & 0 & -1 \\
6 & 0 & 1 & -1
\end{array}\right) ; \quad \begin{aligned}
& v_{0}=6 \\
& v_{1}=1 \\
& v_{2}=5 \\
& v_{3}=13 .
\end{aligned}
$$

Next, $P_{s}=P_{2}$ is determined by

$$
\beta_{0} P_{s}=\beta_{0} P_{2}=\max _{j \neq 0} \beta_{0} P_{j}=6>0
$$


so that the entries $v_{i}=\beta_{i} P_{s}$ (given above) can now be computed. The column $r$ to be dropped from the basis is determined by forming the lexicographic minimum of the vectors (See $\S 2$ ).

$$
\frac{1}{v_{r}} \beta_{r}=\frac{1}{5} \beta_{2}=\min _{v_{i}>0, i \neq 0} \text { (lexico.) } \frac{1}{v_{i}} \beta_{i}
$$

Drop col $r=2$; that is, $P_{7}$.

1st Iteration. The next basis $B^{*}=B_{1}$ is $\left[P_{0}, P_{1}, P_{2}, P_{8}\right]$. To obtain its inverse set: $\beta_{i}^{1}=\beta_{i}-\left(v_{k} / v_{r}\right) \beta_{r}, \quad(k \neq r)$ and $\beta_{r}^{1}=\left(1 / v_{r}\right) \beta_{r}$ where $r=2$ where the superscript (in place of ${ }^{*}$ ) refers to the basis $B=B_{k}$.

$$
B_{1}^{-1}=\left(\begin{array}{l}
\beta_{0}^{1} \\
\beta_{1}^{1} \\
\beta_{2}^{1} \\
\beta_{3}^{1}
\end{array}\right)=\left(\begin{array}{rrrr}
\frac{18}{5} & -\frac{6}{5} & 0 & +\frac{1}{5} \\
\frac{3}{5} & -\frac{1}{5} & 0 & +\frac{1}{5} \\
\frac{2}{5} & \frac{1}{5} & 0 & -\frac{1}{5} \\
\frac{4}{5} & -\frac{13}{5} & \frac{5}{5} & \frac{8}{5}
\end{array}\right) ; \quad \begin{gathered}
v_{0}=\frac{12}{5} \\
v_{2}=\frac{7}{5} \\
v_{3}=\frac{36}{5}
\end{gathered}
$$

where $P_{s}=P_{5}$ is determined by

$$
\beta_{0}^{1} P_{s}=\beta_{0}^{1} P_{5}=\max _{j \neq 0} \beta_{0}^{1} P_{j}=\frac{12}{5}>0
$$

and $P_{j_{r}}=P_{j_{3}}=P_{8}$ is determined by

$$
\frac{1}{v_{r}} \beta_{r}^{1}=\frac{5}{36} \beta_{3}^{1}=\min _{v_{i}>0, i \neq 0}(\text { lexico. }) \frac{1}{v_{i}} \beta_{i}^{1} .
$$

2nd (Final) Iteration.

$$
B_{2}=\left(\begin{array}{cccc}
P_{0} & P_{1} & P_{2} & P_{5} \\
0 & 1 & 1 & 1 \\
-1 & 4 & 3 & 2 \\
-1 & 0 & 7 & 2 \\
-1 & 6 & 0 & 6
\end{array}\right) ; B_{2}^{-1}=\left(\begin{array}{l}
\beta_{0}^{2} \\
\beta_{1}^{2} \\
\beta_{2}^{2} \\
\beta_{3}^{2}
\end{array}\right)=\left(\begin{array}{cccc}
\frac{50}{15} & -\frac{5}{15} & -\frac{5}{15} & -\frac{5}{15} \\
\frac{16}{36} & \frac{11}{36} & -\frac{7}{36} & -\frac{4}{36} \\
\frac{16}{36} & \frac{2}{36} & \frac{2}{36} & -\frac{4}{36} \\
\frac{4}{36} & -\frac{13}{36} & \frac{5}{36} & \frac{8}{36}
\end{array}\right) ;
$$

where no $P_{s}$ can be determined since $\beta_{0}^{2} P_{j} \leqq 0$ for $j \geqq 1$. Thus an optimal 
solution has been obtained (from top row) $\hat{x}_{1}=5 / 15, \hat{x}_{2}=5 / 15, \hat{x}_{3}=5 / 15$ and (from first column) $\hat{y}_{1}=16 / 36, \hat{y}_{2}=16 / 36, \hat{y}_{5}=4 / 36$ where all other $\hat{y}_{i}=0$. The "value of the game" (from upper left corner) is $\hat{x}_{0}=\hat{y}_{0}=$ $50 / 15$. It will be noted that actually $\xi_{0}^{2} P_{j}=0$ for all $j \geqq 1$, which means there exist other bases and corresponding solutions. Williams shows in his book, in all, eight such solutions.

\section{REFERENCES}

1. G. B. Dantzig, A. Orden, and P. Wolfe, "The generalized simplex method," The RAND Corporation, Research Memorandum RM-1264, 1954.

2. Robert Dorfman, "Application of the simplex method to a game theory problem," Activity Analysis of Production and Allocaticn, T. C. Koopmans (Editor), John Wiley and Sons, New York, 1951.

3. Melvin Dresher (and others), Mathematical theory of zero-sum two-person games with finite number or a continuum of strategies, The RAND Corporation, R-115, 1948.

4. H. W. Kuhn, Lectures on the theory of games, reprint, Princeton University Department of Mathematics, 1952.

5. J. C. C. McKinsey, Introduction to the theory of games, McGraw-Hill, New York, 1952.

6. J. von Neumann and Oskar Morgenstern, Theory of games and economic behavior, 2nd Edition, Princeton University Press, 1947, p. 153.

7. Herman Weyl, "Elementary proof of a minimax theorem due to von Neuman," Contributions to the Theory of Games, 1, H. W. Kuhn and A. W. Tucker (Editors), Princeton University Press, 1950.

8. J. D. Williams, The Compleat Strategyst, McGraw-Hill, New York, 1954.

The RAND CORPORATION 



\section{PACIFIC JOURNAL OF MATHEMATICS}

\section{EDITORS}

H. L. Royden

Stanford University

Stanford, California

E. HewitT

University of Washington

Seattle 5 , Washington
R. P. Dilworth

California Institute of Technology Pasadena 4, California

E. G. Straus

University of California

Los Angeles 24, California

\section{ASSOCIATE EDITORS}

E. F. BECKENBACH

C. E. BURGESS

H. BUSEMANN

H. FEDERER

\author{
M. HALL \\ P. R. HALMOS \\ V. GANAPATHY IYER \\ R. D. JAMES
}

M. S. KNEBELMAN

I. NIVEN

T. G. OSTROM

M. M. SCHIFFER
J. J. STOKER

G. SZEKERES

F. WOLF

K. YOSIDA

\section{SUPPORTING INSTITUTIONS}

UNIVERSITY OF BRITISH COLUMBIA

CALIFORNIA INSTITUTE OF TECHNOLOGY

UNIVERSITY OF CALIFORNIA

MONTANA STATE UNIVERSITY

UNIVERSITY OF NEVADA

OREGON STATE COLLEGE

UNIVERSITY OF OREGON

UNIVERSITY OF SOUTHERN CALIFORNIA
STANFORD UNIVERSITY

UNIVERSITY OF UTAH

WASHINGTON STATE COLLEGE

UNIVERSITY OF WASHINGTON

AMERICAN MATHEMATICAL SOCIETY CALIFORNIA RESEARCH CORPORATION HUGHES AIRCRAFT COMPANY 


\section{Pacific Journal of Mathematics}

\section{Vol. 6, No. $1 \quad$ November, 1956}

David Blackwell, An analog of the minimax theorem for vector payoffs..... 1

L. W. Cohen, A non-archimedian measure in the space of real

sequences ..................................... 9

George Bernard Dantzig, Constructive proof of the Min-Max theorem ..... 25

Jim Douglas, On the numerical integration of quasilinear parabolic

differential equations ............................... 35

James Michael Gardner Fell, A note on abstract measure ............. 43

Isidore Isaac Hirschman, Jr., A note on orthogonal systems . . . . . . . . . . 47

Frank Harary, On the number of dissimilar line-subgraphs of a given

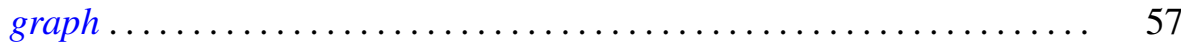

Newton Seymour Hawley, Complex bundles with Abelian group......... 65

Alan Jerome Hoffman, Morris Newman, Ernst Gabor Straus and Olga

Taussky, On the number of absolute points of a correlation ...........

Ernst Gabor Straus and Olga Taussky, Remark on the preceding paper.

Algebraic equations satisfied by roots of natural numbers . . ........ 97

Ralph D. James, Summable trigonometric series ................. 99

Gerald R. Mac Lane, Limits of rational functions . . . . . . . . . . . . . . . 111

F. Oberhettinger, Note on the Lerch zeta function ................. 117

Gerald C. Preston, On locally compact totally disconnected Abelian groups and their character groups ........................... 121

Vikramaditya Singh and W. J. Thron, On the number of singular points, located on the unit circle, of certain functions represented by

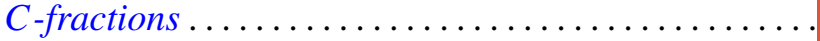

Sherman K. Stein, The symmetry function in a convex body ... 145 Edwin Weiss, Boundedness in topological rings.............

Albert Leon Whiteman, A sum connected with the series for the partition

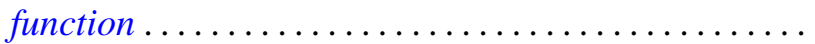

Alfred B. Willcox, Some structure theorems for a class of Banach algebras.

Joseph Lawrence Zemmer, Some remarks on p-rings and their Boolean geometry... 